\title{
Collagen sponge and rhBMP-2 improve socket healing in rats treated with zoledronic acid
}

\section{Ji-Su OH(a) \\ Su-Gwan KIM(a) \\ (a) Chosun University, School of Dentistry, Department of Oral and Maxillofacial Surgery, 375, SeoSukDong, DongGu, Gwangiu 501-759, Republic of Korea.}

Declaration of Interests: The authors certify that they have no commercial or associative interest that represents a conflict of interest in connection with the manuscript.

\section{Corresponding Author:}

Su-Gwan Kim

E-mail:sgckim@chosun.ac.kr

hitps://doi.org/10.1590/1807-3107bor-2017.vol31.0099

Submitted: May 03, 2017

Accepted for publication: Oct 10, 2017

Last revision: Oct 24, 2017

\begin{abstract}
The aim of the present study was to evaluate the possible use of a commercial absorbed collagen sponge and bone morphogenetic protein (BMP) for the prevention of bisphosphonate-related osteonecrosis of the jaw (BRONJ) in rats. Twenty rats received intraperitoneal injections of $0.1-\mathrm{mg} / \mathrm{kg}$ of zoledronic acid three times a week for eight weeks before the extraction of both maxillary first molars after eight weeks. A collagen sponge (experimental group 1) and a collagen sponge with recombinant human BMP-2 (experimental group 2) were applied to the right extraction sockets of ten rats each. The 20 left extraction sockets (control groups 1 and 2) were left unprotected. After eight weeks, all rats were euthanized. Macroscopic analysis, micro-computed tomography (CT) analysis, and histological analysis were performed. There was a significant difference in the bone density between the control and experimental groups on micro-CT analysis. Impaired healing of the extraction sockets, indicating BRONJ, was observed in $80 \%$ of control group 1, $90 \%$ of control group 2, 30\% of experimental group 1, and $20 \%$ of experimental group 2 . The collagen sponge with/without BMP used for protecting the extraction socket had the potential for a positive effect in reducing the incidence of bisphosphonate-related osteonecrosis of the jaw in rats.
\end{abstract}

Keywords: Bone and Bones; Bisphosphonate; Osteonecrosis; Tooth Extraction.

\section{Introduction}

Medication-related osteonecrosis of the jaw (MRONJ) is a well-known complication of antiresorptive or antiangiogenic therapies of the maxilla and mandible. ${ }^{1}$ A special committee of the American Association of Oral and Maxillofacial Surgeons (AAOMS) recommended the nomenclature MRONJ instead of bisphosphonate-related osteonecrosis of the jaw (BRONJ). ${ }^{1}$ It is a medication-related side-effect associated with the antiresorptive therapy for malignant and benign bone diseases with bisphosphonates, bevacizumab, and denosumab. ${ }^{2}$ This has a significant influence on the patients' quality of life, ${ }^{3}$ although a small percentage of patients develop osteonecrosis of the jaw spontaneously, ${ }^{4,5}$ Osteonecrosis of the jaw associated with antiresorptive agents has also been named antiresorptive agent-related osteonecrosis of the jaw (ARONJ). ${ }^{6}$ There is 
still a lack of information concerning the incidence, pathogenesis, treatment strategies, and prevention of MRONJ, although many investigations have been performed ${ }^{5,7}$ since Marx' first report of a patient with BRONJ in 2003. ${ }^{8}$

If all three of the following characteristics are present, the condition is defined as MRONJ: ${ }^{1}$ a) current or previous treatment history with antiresorptive or antiangiogenic agents, b) exposed bone or bone with intra- or extraoral fistula in the maxillofacial region persisting for longer than 8 weeks, and c) no history of radiation therapy for, or obvious metastatic disease in, the jaws.

Mechanical trauma to the oral cavity, including the bone, such as extraction of teeth, implant placement, periodontal surgery, or other dentoalveolar procedures, is a major risk factor for the development of MRONJ. ${ }^{1,3,7,9,10,11}$ Among these invasive dental treatments, tooth extraction is the most common and strongest predisposing factor. ${ }^{1,5,11,12}$ Clinical studies have shown that an extraction is the cause of $40-80 \%$ of cases and thus has a significant association with MRONJ. ${ }^{11,13}$ Hence, many in vivo studies have focused on the healing of extraction sockets in studies related to MRONJ. $9,10,11,12,14,15,16$

The treatment of MRONJ is challenging, ${ }^{14}$ hence, prevention is crucial for its management. ${ }^{2}$ Furthermore, the AAOMS promoted animal studies to validate improved strategies for the prevention of MRONJ as one of its future research areas. ${ }^{1}$ Moreover, the Japanese Allied Committee reported that an understanding of the effects of antiresorptive agents at a cellular level was needed, and that these agents play a critical role in the closure of tooth extraction sockets to protect the alveolar bone from exposure to the oral cavity. ${ }^{6}$ Various animal studies have been reported recently, ${ }^{9,14,17,18}$ but few studies on the prevention methods for MRONJ have been conducted in animal models. The authors attempted to address two questions, based on the collagen sponge, which has been commonly used for socket preservation, and bone morphogenetic protein (BMP), which has been very effective for bone regeneration in the clinic: Does closure of the extraction socket with a collagen sponge have a positive effect on the prevention of alveolar bone exposure? Given that BMP facilitates early resolution of MRONJ, can it also prevent the development of MRONJ?

Bisphosphonate used as an antiresorptive agent in most animal model studies induced osteonecrosis; hence, bisphosphonate was used in this study, and thus, the nomenclature of BRONJ was used. The aim of the present study was to assess whether the use of a commercial absorbed collagen sponge and BMP helps prevent the development of osteonecrosis of the jaw; this was evaluated by observation of the healing of extraction sockets after establishment of the environment of the jaws through tooth extraction in rats to which a high dose of bisphosphonate was administered.

\section{Methodology}

\section{Animals and study design}

All animal experiments were conducted under the auspices of the Chosun University Institutional Animal Care and Use Committee. Twenty female Sprague-Dawley rats (age, 10 weeks; weight, 200-250 g) were provided by DBL Korea. The rats were kept in a specific pathogen-free environment at an ambient temperature of $22 \pm 2^{\circ} \mathrm{C}$ with a humidity of $60 \pm 5 \%$ under a 12-hour light-dark cycle.

The 20 rats were randomly divided into two groups: the collagen sponge group and the collagen sponge with BMP group. Both groups were further divided into subgroups: control groups 1 and 2 (extraction alone; left side) and experimental groups 1 (collagen sponge alone) and 2 (collagen sponge with BMP) (right side). All rats received intraperitoneal injection of $0.1-\mathrm{mg} / \mathrm{kg}$ zoledronic acid (Zometa ${ }^{\circledR}$, Novartis Pharma, Basel, Switzerland) three times a week for eight weeks, for a total of 24 times before extraction, according to previous animal model studies. ${ }^{4}$

\section{Surgical procedure}

After eight weeks of administration of zoledronic acid, all rats underwent a surgical procedure for tooth extraction in accordance with aseptic protocols. Sedation was induced with intramuscular administration of Zoletil ${ }^{\circledR}$ (Virbac, Carros, France, $15 \mathrm{mg} / \mathrm{kg}$ ) along with xylazine (Rumpun ${ }^{\circledR}$, Bayer, Korea), with the recommended doses according to 
the weight of each animal. Local anesthesia with $2 \%$ lidocaine containing 1:100,000 epinephrine was administered before tooth extraction. After the extraction of both maxillary first molars, the sockets were cleaned with sterile normal saline. Type I bovine absorbed collagen (Collaplug, Zimmer Dental Inc., Carlsbad, CA, USA) was applied to the right extraction sockets of 10 rats, and recombinant human BMP-2 (rhBMP-2) (Novosis, Daewoong Pharma, Seoul, South Korea) with type I bovine absorbed collagen as the carrier was applied to the right extraction sockets of 10 rats. The left extraction sockets were left untreated. All extraction sockets were sutured to retain the collagen sponges in the sockets.

\section{Micro-computed tomography (CT) analysis and $H \& E$ analysis}

All rats were euthanized with an anesthetic overdose for tissue harvesting eight weeks after the extractions. The maxillae, including the extraction sites, were dissected and fixed immediately in $10 \%$ buffered formaldehyde. The bone samples were examined by means of a micro-CT scanner (TVXIMT225CT, 225 Kv, Techvalley, Seongnam, Korea) for analysis of the integrity of the alveolar sockets and the presence of osteolysis. The mean bone density values of each slice were measured with image editing software (Adobe Photoshop ${ }^{\circledR}$ CC, Adobe Systems Inc., San Jose, CA, USA) after being converted into 8-bit data (256 greyscale level). Five areas of the extraction socket were selected as the regions of interest. The bone sample was decalcified with $10 \%$ ethylenediaminetetraacetic acid (EDTA) for four months. After being embedded in paraffin, the samples were stained with hematoxylin and eosin (H\&E) for histological analysis. Impaired healing was defined in the present study as the combined findings of the presence of non-vital bone with/without inflammatory cells or bone exposure with no continuity of the epithelium above the extraction socket.

\section{Statistical analysis}

The bone density values were analyzed by the Wilcoxon signed-rank test and the Mann-Whitney test with SPSS Statistics 20 (IBM; Armonk, NY, USA), with a significance level of $5 \%$. The histologic data were analyzed by Fisher's exact test with SPSS 20.0 (Statistical Package for the Social Sciences, IBM, Chicago, IL, USA) with a significance level of $5 \%$.

\section{Results}

Impaired healing of the mucosa in the extraction sockets was observed in a few cases in the experimental groups and in most cases in the control groups, although signs of inflammation, such as pus discharge, swelling, and redness of the mucosa around the extraction socket, were not found. Severe signs of BRONJ were not observed; impaired healing of the extraction socket eight weeks post-extraction might have been a sign of BRONJ.

In most cases, micro-CT analysis revealed explicit delayed bone healing with radiolucency in the extraction sockets in the control groups. In contrast, almost complete normal bone regeneration was observed in the experimental groups. Although extensive bone destruction beyond the extraction socket was not observed, there was a distinct difference in the bone density between the control and experimental groups (Figure 1). There was no significant difference between the experimental groups regarding the greyscale levels $(p>0.05)$, although the greyscale levels of the experimental groups were significantly higher than those of the control groups $(\mathrm{p}<0.05)$ (Table).
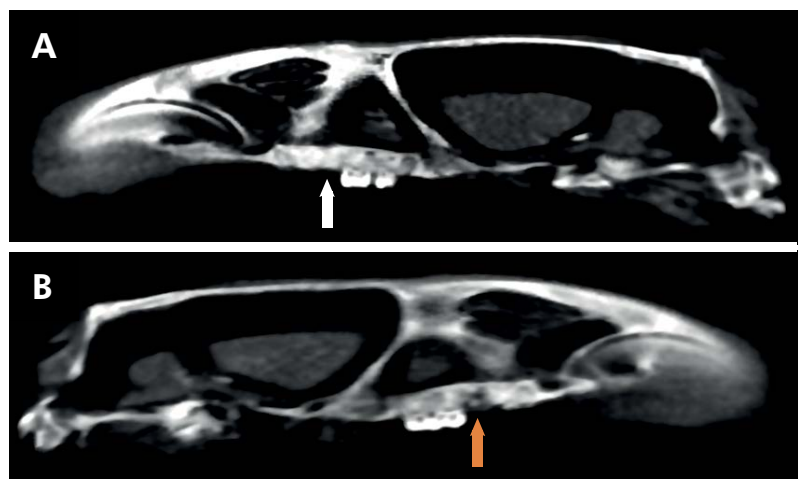

Figure 1. Micro-tomographic scan along sagittal plane of the skull in a rat. Micro- computed tomography features of alveolar socket healing. A (white arrow): showing almost complete mineralization in the experimental group. B (yellow arrow): showing a non-mineralized defect in the control group. 
Experimental group 1 (7/10; 70\%), experimental group 2 (8/10; $80 \%)$, control group $1(2 / 10 ; 20 \%)$, and control group $2(1 / 10 ; 10 \%)$ demonstrated normal bone healing of the extraction sockets, with no signs of bone necrosis, complete re-epithelialization, and well-organized connective tissue. However, impaired bone remodeling was observed with epithelial discontinuity, infiltration of inflammatory cells, and the presence of non-vital bone in most cases in the control groups (85\%), and in only a few cases in the experimental groups (experimental group 1, $30 \%$ and experimental group 2, 20\%, respectively) (Table, Figures 2, 3). The histological results showed a significant difference between the control groups and experimental groups $(p<0.05)$. In contrast, there were no statistically significant differences between experimental groups 1 and 2 regarding the healing of the extraction socket $(\mathrm{p}>0.05)$ (Table).

\section{Discussion}

Bisphosphonates are the most widely used agents for the treatment of osteoporosis and osteopenia. ${ }^{19}$ Moreover, bisphosphonates are used in the treatment or prevention of some pathologic conditions, such as hypercalcemia, Paget's disease, bone metastasis from solid tumors, and multiple myeloma. ${ }^{13,19}$

Bisphosphonates are stable endogenous analogs of inorganic pyrophosphate and are divided into the following two classes according to their chemical structures and mechanisms of action: non-nitrogen-containing bisphosphonates, such as etidronate, tiludronate, and clodronate (low potency), and nitrogen-containing bisphosphonates, such as alendronate, risedronate, ibandronate, and zoledronate, which have high potency in the treatment of osteolytic disorders ranging from osteoporosis to

Table. Summary of micro-CT and histologic analyses eight weeks after extraction in rats injected with zoledronic acid.

\begin{tabular}{lccc}
\hline Groups & Bone density (greyscale levels) & $\begin{array}{c}\text { Non-vital bone W/O } \\
\text { inflammatory cells }\end{array}$ & $\begin{array}{c}\text { Bone exposure with epithelial } \\
\text { impairment }\end{array}$ \\
\cline { 2 - 4 } & Mean values / SD & No. No. & $7^{*}$ \\
\hline Control 1 & $111.20 / 14.55^{\mathrm{a}}$ & $8^{*}$ & $0^{*}$ \\
Experimental 1 & $167.50 / 13.56^{\mathrm{a}}$ & $3^{*}$ & $6^{* *}$ \\
Experimental 2 & $108.40 / 10.62^{\mathrm{b}}$ & $9^{* *}$ & $0^{* *}$ \\
\hline
\end{tabular}

Control 1 and 2, extraction only; experimental 1: insertion of collagen sponge into the extraction socket; experimental 2: insertion of collagen sponge with BMP into the extraction socket; W/O: with/without; SD: standard deviation; No.: number of rats; $a$, b: significant difference; Wilcoxon signed-rank test, $\mathrm{p}<0.05{ }^{*}{ }^{* *}$ : significant difference; Fisher's exact test, $\mathrm{p}<0.05$.
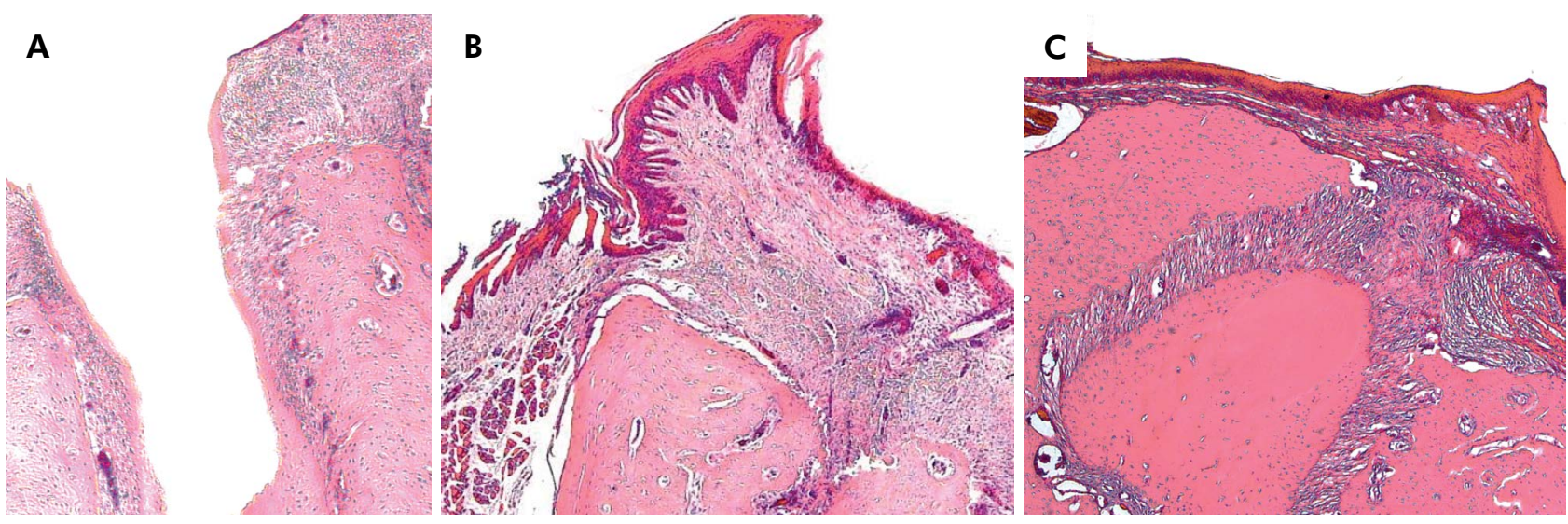

Figure 2. Histologic findings of tooth socket after eight weeks. A: Impaired healing of the epithelium and bone exposure in extraction sockets in the control group. Almost completely healed epithelium in the extraction socket and normal bone remodeling were observed in experimental group 1 (B) and experimental group 2 (C). Hematoxylin-eosin stain, $x 40$. 

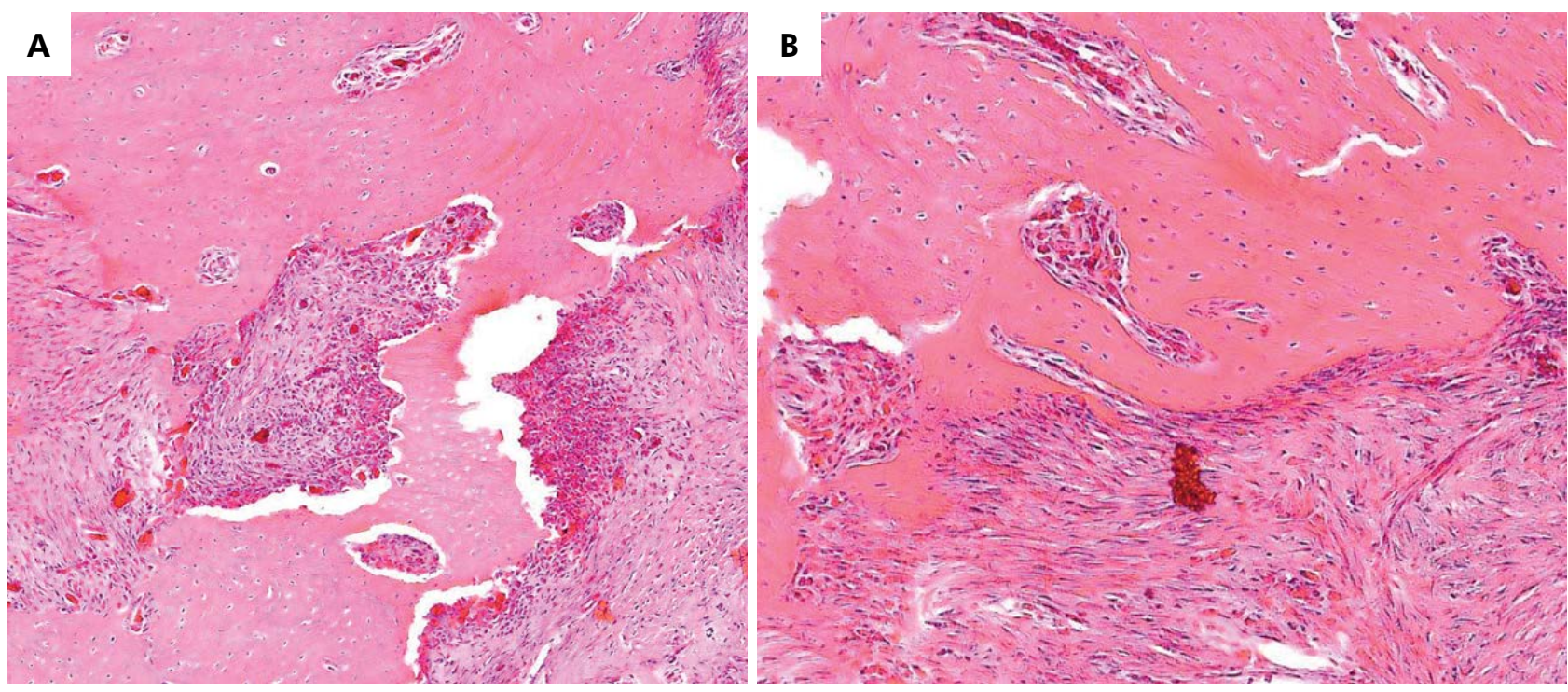

Figure 3. Histologic findings of tooth socket after eight weeks. A: Infiltration of inflammatory cells with non-vital bone was observed in the control group. B: Normal bone remodeling was observed with formation of a new blood vessel in the experimental group. Hematoxylin-eosin stain, x100.

bone metastasis. ${ }^{13,15,20}$ The two most potent nitrogencontaining bisphosphonates are zoledronate and alendronate. ${ }^{20,21}$ Zoledronic acid is the treatment of choice for metastatic breast and prostate cancers and is frequently associated with BRONJ. ${ }^{13,22}$ It was used in this study because of its highest potency among bisphosphonates (its potency is 20 times that of alendronic acid); hence, it is associated with the greatest risk for BRONJ. For the same reason, zoledronic acid is used in almost all animal studies for inducing BRONJ.13, 22,23,24

BRONJ is one of the most serious and well-known potential complications of long-term bisphosphonate therapy. ${ }^{25}$ The treatment for BRONJ poses a challenge to oral and maxillofacial surgeons, pathologists, and oncologists. ${ }^{25}$

Many hypotheses regarding the pathogenesis of BRONJ - including toxicity to the oral epithelium, altered wound healing, high turnover of the bone cells of the jaw, oral biofilm formation, infection and inflammation, antiangiogenesis, suppression of bone turnover, changes in immune surveillance, and osteoblast death - have been proposed. .,16,21,26 $^{2}$ Its etiopathogenesis is multifactorial, ${ }^{27}$ and decreased bone turnover, antiangiogenic effects, and infection are the main hypotheses suggested for the pathogenesis of BRONJ to date. ${ }^{6,28}$ Injury to the alveolar bone, such as tooth extraction, induces the release of bisphosphonates and disrupts epithelial healing. ${ }^{7}$ Despite numerous efforts to develop experimental studies, the etiology and pathophysiology of BRONJ remain largely unclear. ${ }^{3,11,21}$ It is difficult to conduct a prospective clinical study of BRONJ, especially when it involves patients who were administered multiple drugs to control tumor growth and related skeletal complications. ${ }^{3}$ Therefore, pre-clinical animal studies are urgently needed to establish the treatment of and preventive strategies for BRONJ. ${ }^{3,5,6}$

Clinical studies on the prevention of BRONJ have been conducted. ${ }^{29,30}$ Bermúdez-Bejarano et al. ${ }^{30}$ reported, by a review of clinical articles, that antibiotic prophylaxis prior to oral surgery was important in avoiding osteonecrosis and promoting proper healing, since the exposure of the bone in the oral cavity led to bacterial colonization of the modified bone after tooth extraction. Furthermore, atraumatic extraction, a mucoperiosteal flap cover to protect the bone, low-level laser therapy, and treatment with autologous plasma-rich growth factor after extraction as a means of covering the defect were also presented as strategies for the prevention of osteonecrosis of the jaw. ${ }^{30}$ However, these preventive 
methods are sometimes unsuccessful. ${ }^{19}$ Dayisoylu et al. ${ }^{31}$ investigated the preventive effect of locally applied sodium bicarbonate. They reported that local application of sodium bicarbonate had positive effects on the prevention of BRONJ, based on the result that BRONJ was observed in none of the rats when sodium bicarbonate was applied. In this study, early protection with a collagen sponge and a collagen sponge with BMP after extraction showed relatively positive results, thought to be similar to those from use of a mucoperiosteal flap cover to protect the bone.

The collagen matrix is a most representative organic polymer, used for bone and periodontal regeneration, often in combination with other grafting materials. ${ }^{32}$ Collagen enriched with growth factors to induce osteogenesis or improve cellular colonization in short time periods effectively promotes cell viability, proliferation, and adhesion. ${ }^{32}$

rhBMP-2 is a multi-functional growth factor, a member of the transforming growth factor beta (TGF- $\beta$ ) superfamily, and has been used widely in clinics since its FDA approval in 2007 for oral and maxillofacial applications. ${ }^{33,34,35}$ BMP-2 can be utilized in various therapeutic interventions, because it plays an important role in bone formation. ${ }^{34}$

BMP-2 with an absorbed collagen sponge is widely used in clinical practice for bone regeneration. ${ }^{33}$ Collagen has been the most extensively used carrier for the delivery of rhBMPs because it is the most abundant non-mineral component of bones. ${ }^{35}$ rhBMP-2 with a collagen sponge carrier has shown potential for enhanced bone formation in periodontal surgery. ${ }^{37}$ Recently, the positive effect of the combined treatment of BMP-2 and platelet-rich fibrin was reported in patients who needed to continue antiresorptive therapy ${ }^{37}$ However, collagen as an rhBMP-2 carrier has several disadvantages. ${ }^{22,35}$ As a scaffold, collagen has

\section{References}

1. Ruggiero SL, Dodson TB, Fantasia J, Goodday R, Aghaloo T, Mehrotra B et al. American Association of Oral and Maxillofacial Surgeons position paper on medication-related osteonecrosis of the jaw--2014 update. J Oral Maxillofac Surg. 2014;72(10):1938-56. https://doi.org/10.1016/i.joms.2014.04.031 mechanical weaknesses: undesired release can occur, and biodegradation is unpredictable and difficult to control, causing kinetic release of the protein. ${ }^{35}$ In the present study, there was no significant difference between the group with rhBMP-2 with an absorbed collagen sponge and the group with an absorbed collagen sponge alone. This was probably because the extraction sockets of the rats were very small, and rhBMP-2 was undesirable because its early release resulted in only a protective function.

\section{Conclusion}

The present study showed that the use of an absorbed collagen sponge with/without BMP for protecting extraction sockets had a potentially positive effect on reduction of the incidence of $\mathrm{BRONJ}$ in rats.

This study was practical in that protection of the extraction socket for the prevention of BRONJ was possible when tooth extraction was inevitable. However, this study had the limitation of a relatively small sample size, and therefore its outcome cannot be applied directly to clinical practice. Moreover, this study only partially represents the condition of $\mathrm{BRONJ}$ in humans, who have differences in anatomy and physiology. It remains unclear whether the results of animal experiments will agree with clinical results; hence, further clinical study is warranted.

\section{Ethics statement}

All animal experiments were approved by the Chosun University Institutional Animal Care and Use Committee (CIACUC2014-A0016).

\section{Acknowledgments}

This study was supported by the research fund of Chosun University, 2015.

\footnotetext{
2. Svejda B, Muschitz C, Gruber R, Brandtner Ch, Svejda Ch, Gasser RW et al. [Position paper on medication-related osteonecrosis of the jaw (MRONJ)]. Wien Med Wochenschr. 2016;166(1-2):68-74. German. https://doi.org/10.1007/s10354-016-0437-2
} 
3. Yang H, Pan H, Yu F, Chen K, Shang G, Xu Y. A novel model of bisphosphonate-related osteonecrosis of the jaw in rats. Int J Clin Exp Pathol. 2015;8(5):5161-7.

4. Biasotto M, Chiandussi S, Zacchigna S, Moimas S, Dore F, Pozzato $G$ et al. A novel animal model to study non-spontaneous bisphosphonates osteonecrosis of jaw. J Oral Pathol Med. 2010;39(5):390-6. https://doi.org/10.1111/j.1600-0714.2009.00878.x

5. Vidal-Gutiérrez X, Gómez-Clavel JF, Gaitán-Cepeda LA. Dental extraction following zoledronate, induces osteonecrosis in rat's jaw. Med Oral Patol Oral Cir Bucal. 2017;22(2):e177-84. https://doi.org/10.4317/medoral.21609

6. Yoneda $T$, Hagino $H$, Sugimoto $T$, Ohta $H$, Takahashi S, Soen S et al. Antiresorptive agent-related osteonecrosis of the jaw: Position Paper 2017 of the Japanese Allied Committee on Osteonecrosis of the Jaw. J Bone Miner Metab. 2017;35(1):6-19. https://doi.org/10.1007/s00774-016-0810-7

7. Zandi M, Dehghan A, Janbaz P, Malekzadeh H, Amini P. The starting point for bisphosphonate-related osteonecrosis of the jaw: alveolar bone or oral mucosa? A randomized, controlled experimental study. J Craniomaxillofac Surg. 2017;45(1):157-61. https://doi.org/10.1016/i.jcms.2016.10.015

8. Marx RE. Pamidronate (Aredia) and zoledronate (Zometa) induced avascular necrosis of the jaws: a growing epidemic. J Oral Maxillofac Surg. 2003;61(9):1115-7. https://doi.org/10.1016/S0278-2391(03)00720-1

9. Kolpakova ME, Zubareva AA, Artamonova TD, Lisovskaya EK, Chefu SG, Yagmurov OD et al. Experimental model of osteonecrosis of the jaw in rats treated with zoledronic acid. Br J Oral Maxillofac Surg. 2017;55(2):156-159. https://doi.org/10.1016/i.bjoms.2016.10.006

10. Jabbour Z, El-Hakim M, Henderson JE, Albuquerque RF Jr. Bisphosphonates inhibit bone remodeling in the jaw bones of rats and delay healing following tooth extractions. Oral Oncol. 2014;50(5):485-90. https://doi.org/10.1016/i.oraloncology.2014.02.013

11. Conte N, Spolidorio LC, Andrade CR, Esteves JC, Marcantonio E Jr. Experimental osteonecrosis: development of a model in rodents administered alendronate. Braz Oral Res. 2016;30(1):e99. https://doi.org/10.1590/1807-3107BOR-2016.vol30.0099

12. Thumbigere-Math $V$, Sabino MC, Gopalakrishnan R, Huckabay S, Dudek AZ, Basu S, et al. Bisphosphonate-related osteonecrosis of the jaw: clinical features, risk factors, management, and treatment outcomes of 26 patients. J Oral Maxillofac Surg. 2009;67(9):1904-13. https://doi.org/10.1016/i.joms.2009.04.051

13. Sarkarat F, Kalantar Motamedi MH, Jahanbani J, Sepehri D, Kahali R, Nematollahi Z. Platelet-Rich Plasma in Treatment of Zoledronic Acid-Induced Bisphosphonate-related Osteonecrosis of the Jaws. Trauma Mon. 2014;19(2):e17196. https://doi.org/10.5812/traumamon.17196
14. Silva ML, Tasso L, Azambuja AA, Figueiredo MA, Salum FG, da Silva VD, et al. Effect of hyperbaric oxygen therapy on tooth extraction sites in rats subjected to bisphosphonate therapy-histomorphometric and immunohistochemical analysis. Clin Oral Investig. 2017;21(1):199-210. https://doi.org/10.1007/s00784-016-1778-3

15. Mada EY, Santos AC, Fonseca AC, Biguetti CC, Neves FT, Saraiva PP, et al. Effects of green tea and bisphosphonate association on dental socket repair of rats. Arch Oral Biol. 2017;75:1-7. https://doi.org/10.1016/i.archoralbio.2016.12.001

16. Kobayashi Y, Hiraga T, Ueda A, Wang L, Matsumoto-Nakano M, Hata $K$, et al. Zoledronic acid delays wound healing of the tooth extraction socket, inhibits oral epithelial cell migration, and promotes proliferation and adhesion to hydroxyapatite of oral bacteria, without causing osteonecrosis of the jaw, in mice. J Bone Miner Metab. 2010;28(2):165-75. https://doi.org/10.1007/s00774-009-0128-9

17. Keskinruzgar A, Bozdag Z, Aras MH, Demir T, Yolcu $U$, Cetiner S. Histopathological effects of teriparatide in medication-related osteonecrosis of the jaw: an animal study. J Oral Maxillofac Surg. 2016;74(1):68-78 https://doi.org/10.1016/i.joms.2015.07.005

18. Jiang L, Shen X, Wei L, Zhou Q, Gao Y. Effects of bisphosphonates on mandibular condyle of ovariectomized osteoporotic rats using micro-ct and histomorphometric analysis. J Oral Pathol Med. 2017;46(5):398-404. https://doi.org/10.1111/jop.12499

19. Zandi M, Dehghan A, Mohammadi-Mofrad A, Amini P, Vahdatinia F. Short-term perioperative teriparatide therapy for the prevention of medication-related osteonecrosis of the jaw: a randomized, controlled preclinical study in rats. J Craniomaxillofac Surg. 2017;45(2):275-80. https://doi.org/10.1016/i.jcms.2016.12.010

20. Beek ER, Cohen LH, Leroy IM, Ebetino FH, Löwik CW, Papapoulos SE. Differentiating the mechanisms of antiresorptive action of nitrogen containing bisphosphonates. Bone. 2003;33(5):805-11. https://doi.org/10.1016/i.bone.2003.07.007

21. DE Ponte FS, Catalfamo L, Micali G, Runci M, Cutroneo G, Vermiglio $G$ et al. Effect of bisphosphonates on the mandibular bone and gingival epithelium of rats without tooth extraction. Exp Ther Med. 2016;11 (5):1678-84. https://doi.org/10.3892/etm.2016.3168

22. Matsumoto MA, Furquim EMA, Goncalves $A$, Santiago-Júnior JF, Saraiva PP, Cardoso CL et al. Aged rats under zoledronic acid therapy and oral surgery. J Craniomaxillofac Surg. 2017;45(5):781-7. https://doi.org/10.1016/i.jcms.2017.02.002

23. Zandi M, Dehghan A, Malekzadeh H, Janbaz P, Ghadermazi K, Amini P. Introducing a protocol to create bisphosphonate-related osteonecrosis of the jaw in rat animal model. J Craniomaxillofac Surg. 2016;44(3):271-8. https://doi.org/10.1016/i.jcms.2015.12.010 
24. Lim SS, Lee B, Kim IS, Hwang SJ. Differential modulation of zoledronate and etidronate in osseous healing of an extracted socket and tibia defect. Oral Surg Oral Med Oral Pathol Oral Radiol. 2017;123(1):8-19. https://doi.org/10.1016/i.0000.2016.08.009

25. Weber JB, Camilotti RS, Ponte ME. Efficacy of laser therapy in the management of bisphosphonate-related osteonecrosis of the jaw (BRONJ): a systematic review. Lasers Med Sci. 2016;31(6):1261-72. https://doi.org/10.1007/s10103-016-1929-4

26. Fournier P, Boissier S, Filleur S, Guglielmi J, Cabon F, Colombel $\mathrm{M}$ et al. Bisphosphonates inhibit angiogenesis in vitro and testosterone-stimulated vascular regrowth in the ventral prostate in castrated rats. Cancer Res. 2002;62(22):6538-44.

27. Martelli SJ, Damian MF, Gomes AP, Schinestsck AR, Silva AER, Vasconcelos ACU. Comparison of effects of zoledronic acid and clodronate on the bone structure: imaginological and histomorphometrical study in vivo. J Oral Pathol Med. 2017;46(8):632-6. https://doi.org/10.1111/jop.12546

28. Silva PG, Ferreira Junior AE, Teofilo CR, Barbosa MC, Lima Júnior RC, Sousa FB et al. Effect of different doses of zoledronic acid in establishing of bisphosphonate-related osteonecrosis. Arch Oral Biol. 2015;60(9):1237-45. https://doi.org/10.1016/i.archoralbio.2015.05.015

29. Bermúdez-Bejarano EB, Serrera-Figallo MA, Gutiérrez-Corrales A, Romero-Ruiz MM, Castillo-de-Oyagüe R, Gutiérrez-Pérez JL et al. Prophylaxis and antibiotic therapy in management protocols of patients treated with oral and intravenous bisphosphonates. J Clin Exp Dent. 2017;9(1):el41-9. https://doi.org/10.4317/jced.53372

30. Poxleitner P, Engelhardt M, Schmelzeisen R, Voss $P$.

The Prevention of Medication-related Osteonecrosis of the Jaw. Dtsch Arztebl Int. 2017114(5):63-9. https://doi.org/10.3238/arztebl.2017.0063
31. Dayisoylu EH, Ungor C, Tosun E, Ersöz S, Kadioglu Duman M, Taskesen $\mathrm{F}$ et al. Does an alkaline environment prevent the development of bisphosphonate-related osteonecrosis of the jaw? An experimental study in rats. Oral Surg Oral Med Oral Pathol Oral Radiol. 2014;117(3):329-34. https://doi.org/10.1016/i.000o.2013.11.490

32. Ceccarelli G, Presta R, Benedetti L, Cusella De Angelis MG, Lupi SM, Rodriguez Y Baena R. Emerging Perspectives in Scaffold for Tissue Engineering in Oral Surgery. Stem Cells Int. 2017;2017:4585401. https://doi.org/10.1155/2017/4585401

33. Huang $H$, Wismeijer D, Hunziker EB, Wu G. The Acute Inflammatory Response to Absorbed Collagen Sponge Is Not Enhanced by BMP-2. Int J Mol Sci. 2017 Feb 25;18(3):E498. https://doi.org/10.3390/ijms18030498

34. Chen D, Zhao M, Mundy GR. Bone morphogenetic proteins. Growth Factors. 2004;22(4):233-41. https://doi.org/10.1080/08977190412331279890

35. El Bialy I, Jiskoot W, Reza Nejadnik M. Formulation, delivery and stability of bone morphogenetic proteins for effective bone regeneration. Pharm Res. 2017;34(6):1152-70. https://doi.org/10.1007/s11095-017-2147-x

36. Coomes AM, Mealey BL, Huynh-Ba G, Barboza-Arguello C, Moore WS, Cochran DL. Buccal bone formation after flapless extraction: a randomized, controlled clinical trial comparing recombinant human bone morphogenetic protein 2/absorbable collagen carrier and collagen sponge alone. J Periodontol. 2014;85(4):525-35. https://doi.org/10.1902/jop.2013.130207

37. Park JH, Kim JW, Kim SJ. Does the addition of bone morphogenetic protein 2 to platelet-rich fibrin improve healing after treatment for medication-related osteonecrosis of the jaw? J Oral Maxillofac Surg. 2017;75(6):1176-84. https://doi.org/10.1016/i.joms.2016.12.005 